\title{
The Paleoclimate Reconstruction Challenge
}

\author{
Caspar Ammann \\ National Center for Atmospheric Research, Boulder, USA; ammann@ucar.edu
}

Detailed understanding of the full range of annual and seasonal climate variability over the past millennium forms an important basis for the interpretation of the observed record, and for gauging the response of the climate system to various forcings. Using different methods and proxy networks, the climate reconstructions available show general similarity in their depiction of large-scale meantemperature evolution, particularly at the decadal to centennial timescale. There are, however, important differences in reconstructions at the interannual and multicentennial to millennial scale. It is unclear whether these differences result from the selection of specific proxy networks, the potential inability of the included proxies to resolve information at all timescales, or the algorithms themselves (National Research Council, 2006). The paleoclimate community needs to establish a protocol for reassessing its methods to rebuild confidence in the reconstruction efforts.

The last millennium Paleoclimate Reconstruction (PR) Challenge-run under the auspices of the PAGES-CLIVAR-Intersection and co-sponsored by the Electric Power Research Institute (EPRI)—will allow us to directly address these concerns and to establish objective reconstruction benchmarks. The idea is to use results from state-of-the-art coupled Atmosphere-Ocean-General Circulation Models (AOGCMs) in both open- and blind-test reconstruction exercises. Individual reconstruction groups (and anyone who would like to participate) will be brought together and issued a small set of realistic pseudo-proxy series and calibrated "instrumental data" drawn from the model output. They will be asked to reconstruct the simulated climate evolution to the best of their technique's ability. By comparing reconstructions with the full, "true" model climates, each group can assess their performance in great detail. A key objective of this project is to document how much of the true climate can be described with the combined set of reconstruction results, to determine which aspects of the overall or regional climate are captured well, and whether important elements are being missed.

Beyond the main goal of improved understanding of the performance of climate reconstruction methods, it is hoped that the PR Challenge will improve ex-

\section{Paleoclimate Reconstruction (PR) Challenge}

Four key components of the PR Challenge:

A Web-Based Open Reconstruction Access Point coordinated with the NOAA World Data Center will serve as the exchange platform for long-term archiving of methods and data.

The Open PR Challenge Intercomparison provides a set of realistic pseudo-proxy series from existing last millennium AOGCM simulations for a standardized validation of individual reconstruction methods, as well as for a cross-method intercomparison. Reconstruction targets are temperature fields, and ideally also moisture and pressure fields, at seasonal resolution if possible.

The Grand PR Challenge is a double-blind reconstruction exercise based on output from a new, coupled simulation with a realistic but unknown forcing history. The challenge is to provide reconstructions of seasonal temperature patterns, as well as moisture and circulation (pressure) changes.

Post-PR Challenge Assessments will coordinate the cross-community (proxy, modeling and statistics) analysis of PR Challenge results in two crucial workshops that aim at:

1) Evaluation: Identifying the current status in reconstructing climate of the last millennium and identification of outstanding critical issues. Formulation of recommendations for future research foci.

2) Climate Model Assessment: Comparison of climate derived from real world proxies with climate reconstructed from simulated pseudo-proxies, to assess the applicability of model frameworks for studying climate variability.

change among the different paleoclimate reconstruction groups and provide a flexible platform for enhanced interaction with the associated disciplines in climate modeling and statistics. The latter might be particularly helpful with regard to a more formal assessment and quantification of uncertainty and regional climate understanding. The results of the PR Challenge will support and steer the community to develop strategies for improving reconstruction methods, so that past climate variations can be better understood.
PR Challenge organizers:

Caspar Ammann

National Center for Atmospheric Research Nicholas Graham

Scripps Institution of Oceanography and $\mathrm{Hy}$ drologic Research Center

Rosanne D'Arrigo

Lamont Doherty Earth Observatory

Thorsten Kiefer

PAGES International Project Office

\section{Reference}

National Research Council 2006: Surface Temperature Reconstructions for the Last 2000 Years, The National Academies Press, Washington. 